\title{
Erratum to: An efficient scheme for multi-party quantum state sharing of an arbitrary multi-qubit state with one GHZ channel
}

\author{
Min Jiang • Daoyi Dong
}

Published online: 26 July 2012

(C) Springer Science+Business Media, LLC 2012

\section{Erratum to: Quantum Inf Process DOI 10.1007/s11128-012-0429-0}

In the original version of this article, unfortunately, the authors missed to include a note. At the end of the third paragraph of the Introduction in the original article, the following note should be added:

The material in this paper was partially presented at the 10th World Congress on Intelligent Control and Automation, July 6-8, 2012, Beijing, China. The details refer to Jiang M., Dong D.: An efficient scheme for multi-party quantum state sharing via GHZ channels, Proceedings of the 10th World Congress on Intelligent Control and Automation, pp. 5122-5126.

The online version of the original article can be found under doi:10.1007/s11128-012-0429-0.

M. Jiang

School of Electronics and Information Engineering, Soochow University, Suzhou 215006, China e-mail: jiangmin03@gmail.com

D. Dong $(\varangle)$

Institute of Cyber-Systems and Control, State Key Laboratory of Industrial Control Technology, Zhejiang University, Hangzhou 310027, China

e-mail: daoyidong@gmail.com

D. Dong

School of Engineering and Information Technology, University of New South Wales at the Australian Defense Force Academy, Canberra, ACT 2600, Australia 